\title{
Developing culturally responsive social, emotional, and behavioral supports
}

\author{
James Cressey \\ Framingham State University, Framingham, Massachusetts, USA
}

\begin{abstract}
Purpose - The purpose of this paper is to illustrate an interdisciplinary system of targeted student supports, drawing from social and emotional learning (SEL), culturally responsive practices (CRP) and positive behavioral interventions and supports (PBIS). While these approaches are not often synthesized in research literature, innovative educators are integrating multiple theories and practices to achieve better outcomes for students. Design/methodology/approach - The paper uses a descriptive, participatory case study method, including quantitative and qualitative data from a three-year period. The author was a participant in the change process, as well as an observer documenting the outcomes. The case study takes place in a Spanish/ English bilingual elementary school.

Findings - PBIS was a strong influence in the three-year systems change process, due in part to the availability of free, research-based tools. The educators adapted PBIS practices to incorporate SEL, and CRP approaches in several instances. Quantitative data show the increase in PBIS implementation fidelity and one student's progress with a targeted intervention. Qualitative data illustrate the creative, interdisciplinary and contextualized adaptations made by the team.

Originality/value - Guidance is available for educators seeking to implement SEL, CRP and/or PBIS approaches in isolation. To a lesser extent, integrated models are emerging in the literature. There is a need for more descriptive, real-world case examples of how these approaches are implemented and adapted in practice. This study provides educators with one example that can offer examples and implications for practice.
\end{abstract}

Keywords Social and emotional learning, Culturally responsive practice

Paper type Research paper

\section{Introduction}

Social and emotional learning (SEL) is a process through which people develop knowledge and skills related to self-awareness, self-management, social awareness, relationship skills and responsible decision making (CASEL, 2017). As SEL receives more attention in the field of education, its principles and practices are evolving through interdisciplinary research. Culturally responsive practices (CRP) (Ladson-Billings, 2014; Gay, 2000) provide an important lens through which educators can implement SEL in effective, equitable ways for students of color. SEL also intersects with the positive behavioral interventions and supports (PBIS) approach in many ways that can be emphasized to provide effective supports for all students (Bear et al., 2015). While practitioners often draw from multiple perspectives such as SEL, CRP and PBIS simultaneously, the research literature provides little guidance for those seeking integrated models of practice. How are these approaches compatible, and where are their areas of divergence? What do realworld practices look like when educators synthesize SEL, CRP and PBIS?

\section{Integrating SEL, CRP and PBIS}

The present study seeks to illustrate an integrated model with roots in SEL, CRP and PBIS approaches. Culturally responsive SEL has been described by Hammond (2014) with

(C) James Cressey. Published in Journal of Research in Innovative Teaching \& Learning. Published by Emerald Publishing Limited. This article is published under the Creative Commons Attribution (CC BY 4.0) licence. Anyone may reproduce, distribute, translate and create derivative works of this article (for both commercial and non-commercial purposes), subject to full attribution to the original publication and authors. The full terms of this licence may be seen at http://creativecommons.org/licences/by/4.0/legalcode

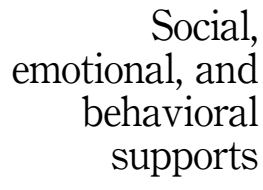

Received 31 January 2019 Accepted 30 March 2019 
JRIT\&L 12,1

specific recommendations for teachers, such as focusing on one's own emotional regulation and triggers through mindfulness. Research in teacher education has advocated for an integrated model of SEL and CRP in the preparation of future teachers (Markowitz et al., 2018; Cressey, 2017). Critiques and challenges are also evident in the examination of SEL through a lens of cultural responsiveness and equity. One state department of education, for example, recently cautioned that "[...] SEL instruction that is not culturally responsive can risk becoming a source of acculturative stress for students who are not members of the dominant group" (DESE, 2019).

The PBIS approach, coming from a different philosophical background in behaviorist theory, provides a promising set of tools for building effective schoolwide systems and creating sustainable change (Solomon et al., 2012). Both SEL and CRP are sometimes described as incompatible with PBIS when certain practices are evaluated. For example, Kozleski (2010) describes positive reinforcement as a non-example of culturally responsive teaching, because it may be confusing to students who are unaccustomed to structures built around adults giving rewards to children. Kohn (1993) and other advocates of SEL have historically discouraged the use of tangible prizes, or even verbal praise, fearing that extrinsic rewards will reduce students' intrinsic motivation. However, more recent recommendations from the fields of SEL and PBIS discuss an integrated approach (Cook et al., 2015). PBIS provides effective tools for behavior change, which can be used to promote SEL competencies such as reflective practices, social awareness, and positive relationships among students and staff (Barrett et al., 2018; NEFC, 2014). These strategies can also be used to promote equity and CRP, when educators work carefully to build culturally responsive PBIS systems (CRPBIS), as described by Bal et al. (2014). The CRPBIS model developed by these authors aims to shift the behavioral goals of the system away from a narrow replacement of unwanted behaviors with target behaviors, and toward a culture of fostering agency and involvement from students in shaping their own school communities. While this model does not explicitly name SEL as an influence, the CRPBIS model includes many practices which align to SEL, such as engaging school communities in rich, open discussions about values, expectations, behaviors and culture. A strong potential remains for future work to integrate SEL, CRP and PBIS, building on these existing efforts.

\section{Targeted support systems}

Beyond the universal, core approach to SEL, CRP and PBIS for all students, it is important to consider the needs of students within the school community who may need additional social, emotional, and behavioral supports. Multi-tiered systems of support (MTSS) are used in schools to offer academic and behavioral supports at graduated levels of need (McIntosh and Goodman, 2016). The MTSS model can be examined in the context of an integrated SEL, CRP and PBIS approach, particularly the use of research-based universal screening tools and targeted interventions. How can we ensure that students who are selected for additional interventions will have an equitable, culturally affirming experience that builds their SEL competencies?

Schoolwide social and emotional screening remains uncommon in schools, despite increasing needs in the population (Lane et al., 2012). Reliable and valid rating scales are available, some of which are brief enough for feasible implementation in schools, and can be obtained at no cost. These include the Student Risk Screening Scale (SRSS) (Drummond, 1994) for externalizing behaviors, and the Student Internalizing Behavior Screener (SIBS) for internalizing behaviors (Cook et al., 2011). Teachers rate each child in their class on 14 items using a 0-3 scale. A team of educators uses the resulting data to select students for additional interventions based on research-based scoring criteria. Absent from much of the universal screening research is an explicit discussion of how to use these tools in a culturally responsive way. Demographically, the school population may not match the sample with 
which the scales were originally researched and normed. Teachers bring qualitative knowledge of each student to the process of data-informed decision making. Educators using a synthesized model of SEL, CRP and PBIS are likely to take a more complex view of data, incorporating quantitative screening results with other information about each child's background, culture, family and school relationships. Does this approach introduce more bias to the screening process, reducing its effectiveness? Or does it afford more contextualized knowledge, improving the validity of the process for each child? These questions remain unresolved when practices from SEL, CRP and PBIS are used simultaneously.

Complex challenges also arise when educators select and adapt research-based interventions to address moderate social, emotional, and behavioral needs. The Check-In, Check-Out (CICO; Crone et al., 2010) pairs students with a staff member who meets with them individually at the beginning and end of each day. Students then check in with their teachers several times a day, using a daily behavior report card to rate their behavior. Extensive research has found CICO to be effective at reinforcing desired behaviors, especially for students who are motivated by adult attention (Wolfe et al., 2016), reflecting the traditional behavioral lens of PBIS. More research is needed to examine culturally responsive CICO practices, and ways to address SEL goals within the use of this intervention. Within the CASEL framework of SEL competencies, one can see how CICO promotes self-awareness through the process of asking students to reflect on their behaviors throughout each day. The intervention also encourages responsible decision making through its focus on positive choices. While these connections between CICO and SEL may be plainly evident to the practitioners implementing the intervention, there is little published scholarly work highlighting these links. Likewise, there is a lack of research investigating $\mathrm{CICO}$ with a culturally responsive approach. What adaptations to CICO might be made for culturally and linguistically diverse school populations, and for individual students, based on CRP? Brown et al. (2018) recommend that educators document their cultural adaptations to evidence-based interventions and track their effectiveness, so these efforts can be replicated. The present study will examine how CICO practices do, in fact, create meaningful opportunities for students to build SEL competencies, and the extent to which the intervention can be used in an equitable, culturally responsive way.

\section{Case study purpose}

This case study aims to provide readers with a clearly articulated example of the multi-year process by which one school team introduced a system for targeted social, emotional, and behavioral supports in a large, high-need, bilingual public elementary school. The study investigates the following question: To what extent did the system change process, and the resulting systems, reflect theories and practices from SEL, CRP and PBIS? The author used a case study method to document the process and evaluate its connections to the theories and research described above. Challenges faced within the system change process will be shared, along with creative solutions and adaptations pursued by the team, and recommendations for future practitioners with similar goals.

\section{Method}

\section{Participants and setting}

The setting for this case study is a large, Spanish/English bilingual K-5 elementary school in an urban/suburban school district in the Northeast. The student body (681 students) is racially and ethnically diverse (69.9 percent Hispanic, 22.5 percent White, 3.7 percent African-American, 3.2 percent Multi-Race, Non-Hispanic, 0.6 percent Asian, and 0.1 percent Native American). English is not the first language for more than half (59.5 percent) of the 
JRIT\&L 12,1

students, and students with disabilities make up 19.5 percent of the student body. The population also has a significant number of students categorized as economically disadvantaged (47.1 percent).

A relevant factor for the context of this study is the school's Spanish/English two-way dual language immersion program. The entire school participates in this instructional model, with the exception of a small, substantially separate special education program made up of a few classrooms. The two-way immersion (TWI) model of bilingual education has three core goals: grade level academic performance, high levels of language and literacy skills in both languages and positive cross-cultural attitudes (Howard, 2000). Research focused on the third goal of TWI programs, and on the related concept of positive cultural identity development, has many implications for the integration of SEL, CRP and PBIS (Howard et al., 2018).

The case study school's mission for students to become "bilingual, bicultural and biliterate" reflects their emphasis on culture as a key feature of their work as educators. It is also notable that this school had established a strong level of universal (tier 1) SEL and PBIS implementation for several years before the case study began. This study examines the process by which the school initiated a system of tier 2 (targeted) supports, but prior to the launch of that endeavor, several years of work went into the development of a universal core PBIS team. For a similar case study outlining the universal tier of PBIS implementation at the school, see Cressey et al. (2015).

\section{Procedures}

The author used a participatory, descriptive case study approach (Reason and Bradbury, 2008 ) in which the subjectivity of the researcher is acknowledged. The author was a change agent who provided consultation to the school staff members throughout the process. The study uses a mixed-methods approach, with quantitative and qualitative data used to achieve its purposes.

Two quantitative measures are reported in the present study. The SRSS and SIBS screening tools described in the literature review above were administered schoolwide, at least twice a year during the case study. The results will include one sample classroom as an illustration of these data. Second, a measure of implementation fidelity was collected using a quantitative scale at three points during the case study. The School-wide PBIS Tiered Fidelity Inventory (TFI) (Algozzine et al., 2014) provides key information about the extent to which PBIS practices are in place and what level of growth can be shown over time.

Qualitative data were gathered through a review of anecdotal records including training materials, meeting agendas, meeting minutes, action plans and documents created by the team. To provide a coherent, process-oriented narrative, the results of the case study will be presented in chronological order. The following results will illustrate the challenges faced, decisions made, and the qualitative themes and quantitative results which the study uncovered over the course of three years.

\section{Results}

\section{Year 1: preparing to build new systems}

The first year of the case study illustrates the extent to which the school staff took their time to develop a thoughtful plan of action, in the hopes of establishing a sustainable system to be introduced the following year. Artifacts from the first year of this process show a strong orientation around PBIS approaches. This is likely due to the foundation of universal, schoolwide PBIS practices which had been established for several years prior. It also reflects the fact that PBIS assessment tools and practice guides are freely available and accessible, and the strength of the PBIS approach in establishing systems change processes that work quickly, and are sustainable in the long term. The PBIS literature recommends a school 
should verify a strong level (80 percent or higher) of universal tier PBIS implementation fidelity before devoting resources to the development of advanced tier systems (Everett et al., 2011). To that end, the Self-Assessment Survey (SAS) (Sugai et al., 2003) was administered yearly to all staff, reaching an average level of 85 percent of PBIS practices in place at the universal tier in the year prior to the start of the present case study. The Schoolwide Evaluation Tool (SET), using interviews with administrators, teachers and students, also showed a moderately strong overall level of PBIS implementation (77 percent total score). The PBIS team felt this was sufficient verification to move forward with the creation of a tier 2 team to generate targeted systems of screening and intervention.

SEL and CRP approaches were also evident in the established practices of the case study school, but perhaps without quantitative measures of implementation fidelity as found in their PBIS work. SEL practices were documented through the use of the Open Circle SEL curriculum, established over ten years ago at the school (Seigle et al., 1997). Training had been provided to teachers, and an internal coach was available on staff, with anecdotal reports indicating a moderate, but inconsistent level of implementation among teachers at the start of the case study period.

CRP were evident through the research-based implementation of the two-way dual language immersion model, established over 20 years ago at the school. As discussed above, this model includes many opportunities for students to explore their own cultural identities, as well as learning about cultures associated with a second language. The case study school has been recognized for exemplary dual language immersion practices several times in years prior to the case study, through the National Association for Bilingual Education, in a textbook case example (Howard et al., 2018), and from the Spanish Ministry of Education.

Effective practices for family engagement also illustrate the school's use of CRP, documented in the results of the Delaware School Climate Survey (Bear et al., 2014) administered annually to all families to assess their views on school climate. Educators and administrators used the results of this survey to confirm areas in which families were satisfied and felt validated, and to select areas for continuous improvement related to school climate and CRP. Based on this established foundation of SEL, CRP and PBIS, the school envisioned a multi-tiered system of supports that would better serve their students.

Forming a systems planning team. Members were chosen from the school support team, a group already in place to discuss students in crisis, to create a new team for systems planning. The school psychologist, two social workers, the school counselor and the principal met with the consultant for a brief presentation about targeted support systems, and to complete a self-assessment to guide their initial priorities. The team completed the tier 2 and tier 3 sections of the Monitoring Advanced Tiers Tool (Horner et al., 2013), the Check-In/Check-Out Self-Assessment (Horner et al., 2005) and the CICO-School-Wide Information System (SWIS) Readiness Checklist (ECS, 2015). In examining the items across all of these tools, the team reflected on features of tier 2 PBIS that were partially in place, such as faculty/staff commitment for CICO, administrator support for CICO, a student identification process for CICO, a CICO point card and access to consultation on using CICO data for decision making. Other features were identified as not currently being in place yet, such as a tier 2 team meeting schedules, roles, routines and processes, team practices to review data regularly, CICO intervention routines, and data entry personnel identified and trained. The team set initial priorities and created an action plan.

The next steps were to establish team roles, expand the membership of the team to include general and special educators, and adopt the CICO-SWIS data tool. The team chose to adopt the name "CARE Advanced Tiers Team" or "CAT Team." They identified possible meeting times, which was an initial roadblock to progress during the winter of this year. 
JRIT\&L 12,1

Figure 1.

Check-in, check-out reflection questions
In the spring, the team began to meet more frequently as new members joined (1-2 meetings per month). The school psychologist and a grade 3 teacher were established as the co-chairs of the team. This paralleled a similar model used in the universal PBIS team in which the school counselor and a grade 4 teacher are co-chairs. Having one classroom teacher paired with a support staff member has proven to be an effective model of shared leadership in this school. The team also discussed other team roles such as notetaker, grade level liaisons and data entry.

To refine their action plan, the CAT team also completed the tier 2 section of the TFI that spring. Items rated as partially in place or not in place were used to generate action items for summer planning, including screening criteria, professional development for faculty and staff, and a student performance data system. One of the next team actions was an effort to secure funding from the school district for team members to work during the summer months.

Intensive summer work. Team members created the CICO daily progress report (DPR) that would be introduced in the fall. The DPR included ratings on each of the four values from the universal school-wide PBIS system (I show CARE for my Classroom, Academics, Respect and Effort). At six points during each day, the teacher would rate the student using a three-level scale ( $2=$ Great!, $1=$ Almost, $0=$ Try Again!). They created a version for younger students with faces to represent each rating. The DPRs were also translated into Spanish. The team also created CICO scripts (Figure 1) for teachers to use in their interactions with students, to ensure the intervention was delivered in a consistent manner. These scripts provide an artifact showing the integration of SEL, CRP and PBIS. Teachers were prompted to ask students reflective questions such as "Does everyone around me feel safe and respected?" This shows a link to the schoolwide PBIS value of "Respect," and also illustrates the SEL practice of prompting students to reflect on their own behaviors. A solely behaviorist approach might be for the teacher to impose an external evaluation, rather than engaging the student in cognitive and emotional reflection within the check-in. These reflective questions also allow the teachers to bring CRP to life through an ongoing evaluation of the classroom climate and an examination of how students contribute to their classroom community. Self-reflection and reflective thinking are key elements of CRP and SEL that were intensified in the CICO protocol.

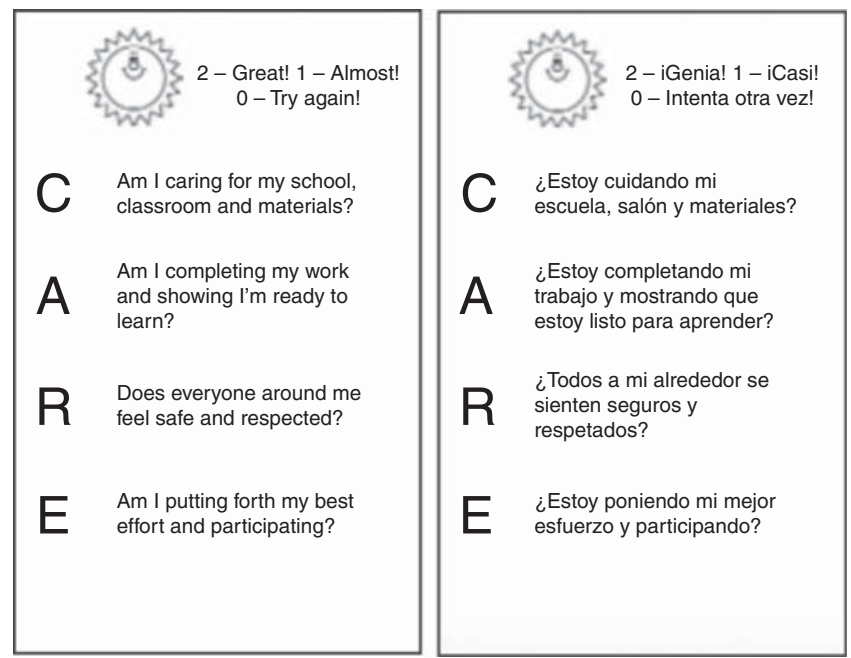


Team members also explored options for universal screening, selecting the SRSS and SIBS tools because they were efficient, no-cost, reliable and valid based on research. Using the school's Google Docs platform, the consultant developed a spreadsheet tool for teachers to quickly enter their screening data. In a hidden tab of the spreadsheet document, a formula was in place to automatically calculate the totals and provide a color-coded summary page for each grade level, viewable only by the CAT team. It was decided that the first screening would take place in mid-October, several weeks into the school year. This would allow teachers some time to get to know their students and observe their behaviors. Meetings would be held after the screening scales were completed, at which time the team members would discuss each student whose results indicated a level of need that could be met by the CICO intervention. CRP is evident in the nuanced approach chosen by the team when working with screening results. They established that the quantitative results of the evidence-based screening tool would be discussed in conjunction with qualitative, personal knowledge of each student. Thus, the team agreed to use an evidence-based tool, but to adapt it using their cultural and individual knowledge of each child.

The consultant also trained the team on the use of the CICO-SWIS data management tool, to be used for progress monitoring. This was an added feature to the school's existing data management system for office discipline referrals, the SWIS (ECS, 2007). The team articulated an integrated approach to using the CICO data as well, acknowledging the limitations of the numerical data and using qualitative knowledge of each child's progress in concert with the quantitative reports.

\section{Year 2: introducing targeted support systems}

As the first year of planning ended, CAT team members were energized and excited by the prospects of launching their new systems. The team co-chairs provided a catalyst for change by working additional hours above and beyond their contractual workloads throughout the late summer and into the fall. In the first weeks of school, the team rehearsed the CICO intervention protocols with two students for several days. This allowed the team to work out logistics of the daily intervention, and to create a training video showing a day in the life of the CICO intervention. The co-chairs were involved in the creation of this video and preparation of a training about universal screening and CICO for all staff members.

Professional development for staff. The CAT team prepared and led a 30-min presentation at the October faculty meeting, providing detailed information about the new universal screening procedure and the CICO intervention. They emphasized CICO as an intervention for the students with mild to moderate needs (not an intensive, individualized intervention for students with more significant needs). The "Day in the Life of CICO" video was shown as another learning modality for staff. The universal screening tool was also presented and a deadline for completion was given.

In response, some teachers expressed concerns about the fact that special education professionals would be used to implement the CICO intervention during the morning and afternoon routines instead of working with students in their classrooms. Others shared their views about the challenges of completing the screening tool by the deadline. To address these issues, the CAT team and principal met to make adjustments. Through creative scheduling, several paraprofessionals were freed up during specific times at the start and end of the day in order to implement CICO without causing major disruptions to their own classroom duties. The universal screening deadline was postponed by a few weeks.

Next, the CAT team chair met with the special education paraprofessionals to train them in delivering the CICO intervention. Most of these new "CICO coordinators" expressed enthusiasm for the opportunity to participate. Coordinators and teachers were provided with the CICO scripts, laminated and ready to clip onto their ID lanyards. 
JRIT\&L 12,1

60

Through discussions with the special education classroom teachers, it was determined that each of the five paraprofessionals could effectively manage up to five students in addition to their classroom duties, allowing for 25 students to participate. Finally, the cafeteria staff were introduced to the CICO program and taught how to provide positive feedback to students through the CICO intervention.

Implementation of universal screening. The SRSS and SIBS screenings were completed by teachers in time for the CAT team to select students for the CICO intervention at their November meeting. Prior to the meeting, one of the co-chairs compiled a list of 39 students who were in the "moderate risk" category on both the internalizing (SIBS) and externalizing (SRSS) scales. Table I shows a sample of results from a third grade classroom. At the meeting, team members held a wide-ranging discussion of each child's strengths and needs based on the screening data as well as their qualitative observations and other sources of information (e.g. office discipline referrals, absences, nurse visits, family contact and teacher comments). The notes from this meeting are artifacts representing how the team foregrounded a CRP lens while analyzing data from a more traditional PBIS/SEL rating scale.

The team decided that several of these students were not a good fit for CICO, based on their knowledge of the students' behavior patterns. Students were not chosen if their behavior had improved in the weeks since the screening was completed, or if team members felt that the student was not motivated by adult attention. This use of a functional behavioral approach reflects the influence of the PBIS model. The team selected 24 students to be offered the CICO intervention. Next, the team offered the intervention to the classroom teachers and solicited their feedback about each student's suitability for the program. If a teacher wished to pursue the intervention with a student, then a consent form would be sent home to the family for their permission. Over the next two weeks, most teachers and families accepted the invitation, with 20 students beginning CICO in December and January.

\begin{tabular}{|c|c|c|c|c|}
\hline Student No. & SRSS total & SRSS risk level & SIBSS total & SIBSS risk level \\
\hline 101 & 9 & High risk & 6 & Moderate risk \\
\hline 102 & 0 & Low risk & 1 & Low risk \\
\hline 103 & 13 & High risk & 6 & Moderate risk \\
\hline 104 & 0 & Low risk & 1 & Low risk \\
\hline 105 & 1 & Low risk & 0 & Low risk \\
\hline 106 & 2 & Low risk & 1 & Low risk \\
\hline 107 & 3 & Low risk & 6 & Moderate risk \\
\hline 108 & 5 & Moderate Risk & 7 & Moderate risk \\
\hline 109 & 0 & Low risk & 0 & Low risk \\
\hline 110 & 1 & Low risk & 0 & Low risk \\
\hline 111 & 0 & Low risk & 0 & Low risk \\
\hline 112 & 0 & Low risk & 0 & Low risk \\
\hline 113 & 3 & Low risk & 1 & Low risk \\
\hline 114 & 3 & Low risk & 13 & High risk \\
\hline 115 & 12 & High risk & 12 & High risk \\
\hline 116 & 12 & High risk & 7 & Moderate risk \\
\hline 117 & 4 & Moderate risk & 7 & Moderate risk \\
\hline 118 & 5 & Moderate risk & 11 & High risk \\
\hline 119 & 11 & High risk & 6 & Moderate risk \\
\hline 120 & 0 & Low risk & 3 & Low risk \\
\hline 121 & 3 & Low risk & 1 & Low risk \\
\hline 122 & 1 & Low risk & 7 & Moderate risk \\
\hline
\end{tabular}

Table I. 120

Fall SRSS and $\quad 121$

SIBS screening results (sample third grade classroom)

Notes: For each scale, $0-3=$ low risk, $4-8=$ moderate risk, $9-21=$ high risk. Students rated at moderate risk on both scales are shown in italics 
Progress monitoring and adaptations. After three weeks, the CAT team met to reflect on their data and make adaptations to the intervention. Their CICO protocol did not include any rewards for students, other than reinforcement through verbal praise. This choice reflects ideas from the SEL mindset as well as the CRP notion of minimizing the use of behavioral modification techniques. However, an adaptation was made for a group of fifth grade students who were provided with an incentive of earning basketball time if they earned 80 percent or more of their daily points. The paraprofessional working with those students suggested the adaptation based on her observations of their greater need for reinforcement, and suggested this basketball time. This practice reflects PBIS strategies often associated with more intensive tiers of intervention, and was reported to be effective for those students by their coordinator. The basketball incentive also engaged students in an activity that was culturally responsive to their interests and strengths, and built their SEL through positive peer relationships.

After several more weeks, nearly all of the students were found to consistently exceed the goal of earning 80 percent of their daily points. Questions arose about how and when to fade out use of the intervention for each student. The team made plans to administer the SRSS and SIBS screening tools a second time, in late February to help with decisions about which students should graduate from CICO, and which new students might be offered the intervention as spaces became available. In March, the team reviewed the February screening results, CICO-SWIS data, and anecdotal information about each child based on their knowledge of the students in class and other settings. For example, they discussed a subgroup of 5th graders who were experiencing stressors in their families and community, and would benefit from support as they prepared for the transition to middle school. This was an example of how the team worked from a mindset reflecting SEL and CRP, to supplement the PBIS approach of using the quantitative data in the screening process. Had they relied solely on the rating scale scores, they may not have offered the CICO intervention to the new group of 5 th graders.

Figure 2 illustrates the progress made by "Jessica" (pseudonym), a third grader who had shown signs of emotional distress, challenges with work completion, and disruptive behaviors in the fall. Jessica exceeded the goal of earning 80 percent or more of her daily points from the start of her intervention. Qualitative observations from her teachers show that Jessica responded well to the opportunities for individual attention and check-ins afforded to her by CICO.

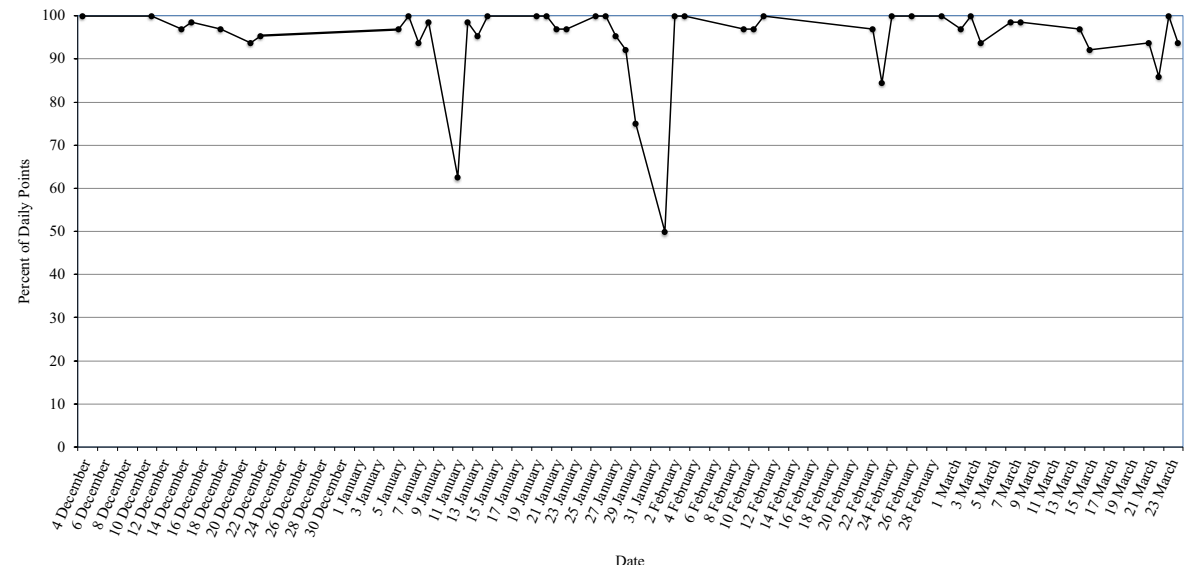

Note: Dates without data (student absences, school closings, or missing data) were removed

Figure 2. Check-in/check-out progress monitoring data for "Jessica" 
JRIT\&L 12,1

62

Over time, she became more skilled at accurately rating her own behavior. Toward the end of the intervention period, teachers began to fade out the adult feedback and allowed her to self-monitor. Jessica was hesitant to discontinue the intervention when the teachers suggested that she "graduate" from CICO, but over time she herself initiated a fading out of the frequency of her check-ins and then was ready to stop them entirely after almost 16 weeks of CICO participation. Jessica's example illustrates key practices from SEL, CRP and PBIS that were integrated by her teachers and support staff. The adults shared control of the decision-making process with Jessica, and recognized her emotional experiences and wishes, not just her compliance with school behavior expectations. Letting Jessica take the lead in her own fading process was an effective way of empowering her responsible decision making, reflecting the integration of SEL and CRP in the decisions of her teachers.

CAT team members discussed how to conclude the CICO program at the end of the year, knowing that most of the students had formed strong emotional connections with their coordinators. Several students had already asked to participate again the following year. The team held celebrations of success for the CICO students with extra recess and certificates of participation. The students also signed cards and wrote thank you letters for their CICO coordinators. These efforts were well received by the students, and the coordinators also responded well to being recognized and thanked by their students. The staff members volunteered to serve as CICO coordinators again in the fall. These choices, focused on relationships and emotional experiences of students and staff, provide more illustrations of how SEL and CRP approaches were brought into a traditional PBIS intervention by the team.

Preparing for Year 3. The next summer, the CAT team received district funding for a 5-hour workshop with the consultant. The team identified action items, guided in part by completing the TFI again in the spring. The TFI showed an improvement in the implementation fidelity of the tier 2 systems in place (see Table II). Within the Teams subscale, action items include continuing to build the capacity of the team through its membership, procedures and roles. The team identified some screening and nomination procedures, but lacked a written request for assistance that teachers can use to make referrals for students who may need a tier 2 intervention. Within the Interventions subscale of the TFI, the team was still in need of improvement in several areas, most notably in developing a menu of research-based interventions beyond CICO. Finally, within the Evaluation subscale of the TFI,

\begin{tabular}{lrrr}
\hline Subscale/item & Year 1 & Year 2 & Year 3 \\
\hline Teams & $25 \%$ & $62 \%$ & $75 \%$ \\
$\quad$ Team composition & 1 & 1 & 1 \\
Team operating procedures & 1 & 1 & 2 \\
Screening & 0 & 2 & 2 \\
Request for assistance & 0 & 1 & 1 \\
Interventions & $30 \%$ & $80 \%$ & $83 \%$ \\
Options for Tier II interventions & 0 & 1 & 1 \\
Tier II critical features & 1 & 2 & 2 \\
Practices matched to student need & 1 & 2 & 2 \\
Access to Tier I supports & 1 & 1 & 2 \\
Professional development & 0 & $62 \%$ & 1 \\
Evaluation & $0 \%$ & 1 & 1 \\
Level of use & 0 & 2 & 2 \\
Student Performance data & 0 & 1 & 1 \\
Fidelity data & 0 & 1 & 2 \\
Annual evaluation & 0 & &
\end{tabular}

Table II. inventory (TFI) Tier 2 items and subscales

Notes: Item scoring criteria: $0=$ not implemented; $1=$ partially implemented; $2=$ fully implemented 
the team reflected on areas for improvement such as gathering more formal implementation fidelity data for the CICO intervention and other tier 2 interventions.

With their workshop time, the team updated their CICO intervention materials with minor adaptations based on feedback they received during the previous year. The team also examined new research providing an alternative to the SIBS for internalizing problems. The SRSS-I5 (Lane et al., 2015) has only five items instead of seven, and has been researched more closely in conjunction with the SRSS. The team decided to use this new internalizing scale and the SRSS screener they had used for externalizing problems.

\section{Year 3: improved systems for screening and intervention}

At the start of year 3, it was determined that the school psychologist would continue as a CAT team co-chair. However, the classroom teacher who served as the second co-chair did not return to her position at the school, so a new grade 3 teacher stepped in to fill that role. Other team members left or joined the team, with a core group of members returning for a second or third year of involvement in the initiative. Screening timelines were earlier in the fall, allowing for a more preventative approach to targeted intervention, while still leaving time for building rich knowledge of individual students. Teachers and paraprofessionals who had participated in CICO during year 2 provided mentoring to colleagues who were new to the intervention. Peer mentoring also became an element of the intervention. Students who had successfully participated in CICO during year 2 were asked to help some of the students to learn the procedures of the intervention early in year 3. This peer mentoring adaptation is another reflection of SEL and CRP that can be found in the practices established at the school. By emphasizing the importance of peer relationships, the staff were helping to promote social competencies and building a positive school culture around the CICO intervention, rather than a negative stigma associated with participation.

The CAT team continued to use the TFI results from year 2 to guide their planning. They worked toward greater implementation fidelity by seeking a more consistent use of data, incorporating multiple measures for screening and nomination beyond the SRSS. They formally tracked school nurse visits. The team also examined ODRs (office discipline referrals) within the SWIS database on a regular basis. Based on recommendations from McIntosh et al. (2010), they looked for students who had earned between two and five ODRs by October, as a possible indicator of need for support.

The TFI indicated the need for the team to develop a broader menu of interventions beyond CICO. They spent several meetings over the course of the year collaborating on this menu, based on existing practices and potential resources available in the school and community. Examples already in place included services provided by the school counselor and social workers, such as social skills groups. Going forward, they sought to establish a systematic process of using data to guide the selection of multiple interventions, based on student need. TFI areas still unmet included fidelity checks on the implementation of interventions, a request form for teachers to seek support for a student, and more professional development for staff members. The team also completed the tier 3 (intensive) subscale of the TFI in the hopes of establishing a more systematic approach to their most intensive interventions.

\section{Discussion}

The present study has provided a narrative, descriptive case example of how SEL, CRP and PBIS approaches can be integrated throughout the process of developing a targeted (tier 2) system of screening and intervention supports. Quantitative data show the potential of this model for strengthening implementation fidelity (through the TFI), for conducting 
JRIT\&L 12,1 meaningful and reliable universal screenings (through the SRSS and SIBS), and for supporting prosocial student behavior and self-reflection skills (through the CICO progress monitoring results). Most of the quantitative data used by this team was indicative of the PBIS approach, but adaptations were made by the school staff to the data analysis procedures and intervention procedures, reflecting the value they place on SEL and CRP as equally important theories and practices. These adaptations, and the use of a case study to document them, supports the recommendations found in previous scholarship about the integration of SEL, CRP and/or PBIS approaches (Brown et al., 2018).

The process also illustrates how the team regularly returned to evidence-based practices for the implementation of a targeted system (Everett et al., 2011), for universal screening (Lane et al., 2012) and for targeted intervention (Crone et al., 2010). While they made adaptations, they continued to ground their work in self-evaluation using the PBIS and SEL research base. This raises implications and questions about how CRP can be better assessed by teams such as this one. What tools might be available to assess the implementation fidelity of CRP in ways that are similar to the TFI and SET? Or could these tools be developed by future research, using quantitative, qualitative or mixed methods of selfassessment of CRP?

These findings also reinforce the value of taking several months to plan a new system before it is introduced. Presenting the system ahead of time allowed staff members to adjust to the new expectations. The participation of general and special education teachers on the CAT team was essential for increasing staff buy-in for the new systems. Having time during the summer was also a key feature of their success, despite the limited amount of time for which they received funding. By planning ahead to maximize these hours in small group work, and inviting a consultant to support them on these days, their summer work was efficient and provided momentum for the start of the school year. Practitioners with similar goals and resources might consider taking even more time to plan their systems, so that more CRP and SEL practices can be intentionally and explicitly discussed by their teams.

\section{Limitations}

The methods and context of this case study prevents broad generalizations from being made from this sample to other settings. Participatory case study methods allowed the author to engage in the process as an insider/outsider, advocating for real change while also studying the outcomes; however, this dual role also must be acknowledged as a source of bias and subjectivity in the study. Investigating an integrated SEL, CRP and PBIS model within the context of a two-way dual language immersion program adds another layer of specificity to these findings. While this brings a limitation to the study's generalizability, it also allowed the author to examine a rich cultural context in which these practices could thrive. Practitioners working under significantly different circumstances may need to make different kinds of adaptations to evidence-based practices to meet the needs of their own communities, as is recommended within the CRP, SEL and PBIS approaches.

\section{Conclusions}

To promote SEL effectively, particularly in high-needs, public school settings, educators face complex challenges and often work with limited resources. However, they may also have a rich cultural base to work from, if the school culture is built on cultural responsiveness and equity. Evidence-based SEL programs can provide a starting place for this work, but may be strengthened through culturally responsive adaptations. To reach and teach a student body that is culturally and linguistically diverse, SEL must be implemented in a culturally responsive and equitable way, or the results may not be supportive for students. Furthermore, when students bring a wide range of social, emotional, and behavioral needs to school, educators must respond with a variety of 
intervention strategies, including targeted and individualized supports. Multi-tiered PBIS research and practices provide another key to this complex work, with powerful and widely available tools for systems change, data collection, intervention and progress monitoring. Without an emphasis on SEL and CRP, these PBIS practices can be used in ways that are oriented predominantly to compliance and obedience. When implemented as an integrated system, with equity and student well-being as long-term goals, these are compatible theories and practices of great value.

\section{References}

Algozzine, B., Barrett, S., Eber, L., George, H., Horner, R., Lewis, T., Putnam, B., Swain-Bradway, J., McIntosh, K. and Sugai, G. (2014), "School-wide PBIS tiered fidelity inventory", OSEP Technical Assistance Center on Positive Behavioral Interventions and Supports, Eugene, OR.

Bal, A., Kozleski, E.B., Schrader, E.M., Rodriguez, E.M. and Pelton, S. (2014), "Systemic transformation from the ground-up: using learning lab to design culturally responsive schoolwide positive behavioral supports", Remedial \& Special Education, Vol. 35 No. 6, pp. 327-339.

Barrett, S., Eber, L., McIntosh, K., Perales, K. and Romer, N. (2018), "Teaching social-emotional competencies within a PBIS framework", OSEP Technical Assistance Center on Positive Behavioral Interventions and Supports, Eugene, OR.

Bear, G., Yang, C., Mantz, L., Pasipanodya, E., Hearn, S. and Boyer, D. (2014), Technical Manual for Delaware School Survey: Scales of School Climate, Bullying Victimization, Student Engagement, and Positive, Punitive, and Social Emotional Learning Techniques, Center for Disabilities Studies, Newark, DE.

Bear, G.G., Whitcomb, S.A., Elias, M.J. and Blank, J.C. (2015), "SEL and schoolwide positive behavioral interventions and supports", in Durlak, J.A., Domitrovich, C.E., Weissberg, R.P. and Gullotta, T.P. (Eds), Handbook of Social-Emotional Learning, Guilford Press, New York, NY, pp. 453-467.

Brown, C., Maggin, D.M. and Buren, M. (2018), "Systematic review of cultural adaptations of schoolbased social, emotional, and behavioral interventions for students of color", Education \& Treatment of Children, Vol. 41 No. 4, pp. 431-455.

CASEL (2017), "Core SEL competencies", Collaborative for Academic, Social, and Emotional Learning, Chicago, IL.

Cook, C.R., Frye, M., Slemrod, T., Lyon, A.R., Renshaw, T.L. and Zhang, Y. (2015), “An integrated approach to universal prevention: independent and combined effects of PBIS and SEL on youths' mental health", School Psychology Quarterly, Vol. 30 No. 2, pp. 166-183.

Cook, C.R., Rasetshwane, K.B., Truelson, E., Grant, S., Dart, E.H., Collins, T.A. and Sprague, J. (2011), "Development and validation of the student internalizing behavior screener: examination of reliability, validity, and classification accuracy", Assessment for Effective Intervention, Vol. 36 No. 2, pp. 71-79.

Cressey, J.M. (2017), "Social-emotional learning in teacher education: a needs assessment survey of teacher educators", Massachusetts Consortium for Social-Emotional Learning in Teacher Education SEL-Ted, Boston, MA.

Cressey, J.M., Whitcomb, S.A., McGilvray-Rivet, S.J., Morrison, R.J. and Shander-Reynolds, K.J. (2015), "Handling PBIS with CARE: scaling up to school-wide implementation", Professional School Counseling, Vol. 18 No. 1, pp. 90-99.

Crone, D.A., Hawken, L.S. and Horner, R.H. (2010), Responding to Problem Behavior in Schools: The Behavior Education Program, 2nd ed., The Guilford Press, New York, NY.

DESE (2019), "Social and emotional learning in Massachusetts", Department of Elementary and Secondary Education, Malden, MA.

Drummond, T. (1994), The Student Risk Screening Scale (SRSS), Josephine County Mental Health Program, Grants Pass, OR. 
JRIT\&L 12,1

ECS (2007), "School-wide information system", Educational and Community Supports, Eugene, OR, available at: www.pbisapps.org (accessed April 15, 2019).

ECS (2015), "CICO-SWIS facilitator certification training”, Educational and Community Supports, Eugene, OR, available at: www.pbisapps.org (accessed April 15, 2019).

Everett, S., Sugai, G., Fallon, L., Simonsen, B. and O'Keefe, B. (2011), "School-wide tier II interventions: check-in check-out getting started workbook", OSEP Center on Positive Behavioral Interventions and Supports, Center for Behavioral Education and Research, University of Connecticut, Storrs, CT.

Gay, G. (2000), "Culturally responsive teaching in special education for ethnically diverse students: setting the stage", International Journal of Qualitative Studies in Education, Vol. 15 No. 6, pp. 613-629.

Hammond, Z. (2014), Culturally Responsive Teaching and the Brain, Corwin, Thousand Oaks, CA.

Horner, R.H., Todd, A. and Dickey, C. (2005), Check-in/Check-out Self-Assessment, Educational and Community Supports, Eugene, OR.

Horner, R.H., Sampson, N.K., Anderson, C.M., Todd, A.W. and Eliason, B.M. (2013), Monitoring Advanced Tiers Tool, Educational and Community Supports, Eugene, OR.

Howard, E. (2000), "Two-way immersion: a key to global awareness", Educational Leadership, Vol. 60 No. 2, pp. $62-64$.

Howard, E.R., Lindholm-Leary, K.J., Rogers, D., Olague, N., Medina, J., Kennedy, D., Sugarman, J. and Christian, D. (2018), Guiding Principles for Dual Language Education, 3rd ed., Center for Applied Linguistics, Washington, DC.

Kohn, A. (1993), Punished by Rewards, Houghton Mifflin Company, Boston, MA.

Kozleski, E. (2010), Culturally Responsive Teaching Matters!, ASU Equity Alliance, Tempe, AZ.

Ladson-Billings, G. (2014), "Culturally relevant pedagogy 2.0: a.k.a. the Remix", Harvard Educational Review, Vol. 84 No. 1, pp. 74-84.

Lane, K.L., Menzies, H.M., Oakes, W.P. and Kalberg, J.R. (2012), Systematic Screenings of Behavior to Support Instruction from Preschool to High School, The Guilford Press, New York, NY.

Lane, K.L., Oakes, W.P., Swogger, E.D., Schatschneider, C., Menzies, H.M. and Sanchez, J. (2015), "Student risk screening scale for internalizing and externalizing behaviors: preliminary cut scores to support data-informed decision making", Behavioral Disorders, Vol. 40 No. 3, pp. 159-170.

McIntosh, K. and Goodman, S. (2016), Integrated Multi-tiered Systems of Support, The Guilford Press, New York, NY.

McIntosh, K., Frank, J.L. and Spaulding, S.A. (2010), "Establishing research-based trajectories of office discipline referrals for individual students", School Psychology Review, Vol. 39 No. 3, pp. 380-394.

Markowitz, N., Thowdis, W. and Gallagher, M. (2018), "Sowing seeds of SEL: university-district partnership builds social and emotional learning across the teacher pipeline", Learning Professional, Vol. 39 No. 4, pp. 30-34.

NEFC (2014), "Responsive classroom and PBIS: Can Schools Use them Together?", Northeast Foundation for Children, Greenfield, MA.

Reason, P. and Bradbury, H. (Eds) (2008), The Sage Handbook of Action Research: Participative Inquiry and Practice, Sage Publications, Los Angeles, CA.

Seigle, P., Lange, L. and Macklem, G. (1997), Open Circle Curriculum., Reach Out to Schools Social Competency Program, The Stone Center, Wellesley College, Wellesley, MA.

Solomon, B.G., Klein, S.A., Hintze, J.M., Cressey, J.M. and Peller, S.L. (2012), "A meta-analysis of schoolwide positive behavior support: an exploratory study using single-case synthesis", Psychology in the Schools, Vol. 49 No. 2, pp. 105-121. 
Sugai, G., Horner, R. and Todd, A. (2003), "EBS self-assessment survey”, Version 2.0, Educational and Community Supports, University of Oregon, Eugene, OR.

Wolfe, K., Pyle, D., Charlton, C.T., Sabey, C.V., Lund, E.L. and Ross, S.W. (2016), “A systematic review of the empirical support for check-in check-out", Journal of Positive Behavioral Interventions, Vol. 18 No. 2, pp. 74-88.

\title{
Further reading
}

Feinauer, E. and Howard, E. (2014), "Attending to the third goal: cross-cultural competence and identity development in two-way immersion programs", Journal of Immersion and Content-Based Language Education, Vol. 2 No. 2, pp. 257-272.

\begin{abstract}
About the author
Dr James Cressey is Associate Professor of Education at Framingham State University. He is a Licensed Special Educator and Nationally Certified School Psychologist. Dr Cressey has worked with children and families since 1999 in many roles including direct care staff, special education teacher, individual therapist, group therapist, family therapist, school psychologist, summer school administrator, consultant and researcher. Dr Cressey's scholarship and teaching are focused on inclusion and equity through evidence-based inclusive practices such as universal design for learning, social and emotional learning, culturally responsive practices, positive behavioral interventions and supports, and multi-tiered systems of support. Dr James Cressey can be contacted at: jcressey@framingham.edu
\end{abstract}

For instructions on how to order reprints of this article, please visit our website: 